Ann. Génét. Sél. anim,. I979, 11 (I), 93-ro3.

\title{
Déterminisme génétique du nombre de cæca pyloriques chez la Truite fario (Salmo Trutta, Linné) \\ et la Truite arc-en-ciel (Salmo Gairdneri, Richardson)
}

\section{III. - Effet du génotype et de la taille des œufs sur la réalisation du caractère chez la Truite fario}

\author{
J. M. BLANC( $\left({ }^{(}\right)$, B. CHEVASSUS $\left({ }^{2}\right)$ et P. BERGOT $\left({ }^{3}\right.$ \\ avec la collaboration de J. J. YANCr( $\left.{ }^{1}\right)$ et Nelly KaUshik ${ }^{(3)}$ \\ (1) Laboratoire d'Écologie des Poissons, \\ Centre de Recherches hydrobiologiques, I.N.R.A., \\ Saint-Pée-sur-Nivelle, 643 Io Ascain \\ (2) Laboratoire de Physiologie des Poissons, \\ Centre national de Recherches zootechniques, I.N.R.A., \\ $7835^{\circ}$ Jouy-en-Josas \\ (3) Laboratoive de Nutrition des Poissons, \\ Centre de Recherches hydrobiologiques, I.N.R.A., \\ Saint-Pée-sur-Nivelle, 64310 Ascain
}

\section{Résumé}

L'analyse de la variation du nombre de cæca pyloriques chez la Truite fario élevée en pisciculture a montré que :

- le sexe des animaux n'a aucune influence significative; mative 0,4 );

- une part importante de variabilité est d'origine génétique additive (héritabilité approxi-

- il existe un effet maternel inhérent à la taille des œufs, les alevins issus de gros ceufs. tendant à avoir des nombres de cæca élevés (corrélation $+0,5$ );

- enfin, l'action conjointe de ce facteur maternel et des autres facteurs génétiques et environnementaux semble comporter des effets interactifs dont l'étude plus approfondie serait utile.

\section{Introduction}

Les cæca pyloriques sont des évaginations de l'intestin antérieur que l'on' rencontre en nombre variable chez de nombreux poissons, notamment les Salmonidés. Ce nombre est fréquemment utilisé comme un critère taxonomique. Il est, par ailleurs, 
susceptible d'avoir une influence sur les caractéristiques digestives, en particulier la vitesse de transit des aliments (BERGOT et al., I975), et sur la croissance (BERGOT et al., 1979).

Dans une publication antérieure (BERGOT et al., I976) nous avons montré qu'à côté des différences interspécifiques utilisées en systématique s'ajoutaient, chez la Truite arc-en-ciel ainsi que chez la Truite fario, une variabilité entre populations de la même espèce ainsi que des effets génétiques intrapopulations importants. Dans une étude ultérieure réalisée chez la Truite arc-en-ciel (Chevassus et al., I979), nous avons obtenu une valeur moyenne de 0,52 pour l'héritabilité indiquant ainsi qu'environ la moitié de la variabilité phénotypique totale était liée à des effets de type génétique additif. Nous avons d'autre part montré que des effets environnementaux comme le milieu d'élevage et à un moindre degré l'alimentation induisent également des fluctuations significatives.

$L_{\text {a }}$ présente étude a pour objet l'estimation de la variabilité génétique de ce caractère chez la Truite fario (Salmo trutta Linné) élevée en pisciculture et l'appréciation de l'effet maternel inhérent à la taille des œufs.

\section{Matériel et méthodes}

Le matériel animal utilisé dans cette analyse a été obtenu à partir d'un élevage expérimental destiné à l'étude des paranètres génétiques de la croissance (BLANC, I979).

\section{Structure de la population expérimentale}

La population expérimentale était constituée de 150 familles représentant 6 plans factoriels complets de 5 mâles par 5 femelles chacun, réalisés en décembre I975 à la pisciculture de Saint-Pée-sur-Nivelle (Pyrénées-Atlantiques). Le choix des reproducteurs (parmi les géniteurs de 3 ans, souche de la pisciculture) ainsi que leur association dans les différents croisements furent faits au hasard. Toutefois, pour des raisons pratiques tenant aux différences de maturation des femelles, les 6 séries ne purent être réalisées simultanément, mais en deux temps à une semaine d'intervalle : le 4 décembre pour les séries I à 3 , et le II décembre pour les séries 4 à 6 .

\section{Conditions d'élevage}

Les œufs fécondés furent incubés sur des clayettes compartimentées (un compartiment par famille) placées dans le même récipient à la température moyenne de $8{ }^{\circ} \mathrm{C}$. Au stade œillé, un échantillon de roo œufs fut prélevé dans chaque famille et volumétré par déplacement d'eau dans une éprouvette de $25 \mathrm{~cm}^{3}$ (précision approximative de la mesure : $\pm 0,2 \mathrm{~cm}^{3}$ ).

Peu avant l'éclosion, les œufs furent transférés dans l'installation d'élevage. Celle-ci se composait d'auges aménagées en 150 compartiments (un par famille) de même taille (fond : $80 \mathrm{~cm} \times 42 \mathrm{~cm}$; hauteur d'eau : I $2 \mathrm{~cm}$ ) et alimentés avec la même eau (eau de la Nivelle filtrée).

Le 29 juillet I976, les alevins ayant atteint une taille moyenne de $6 \mathrm{~cm}$, 
5 individus furent prélevés au hasard dans chaque famille et sacrifiés ( $75^{\circ}$ au total). Sur chacun on déterminera le sexe, la longueur, le poids et le nombre de cæca pyloriques.

\section{Méthodes statistiques}

Les résultats que nous présentons ne concernent que les données relatives au nombre de cæca et à la taille des œufs. L'étude de la distribution du nombre de cæca nous a conduit à faire les analyses sur la variable transformée $\mathrm{Y}=\operatorname{I00} \log _{10} \mathrm{X}$. Les volumes d'œufs mesurés, étant répartis sensiblement normalement, n'ont fait l'objet d'aucune transformation.

Nous avons utilisé les modèles d'analyse de variance suivants :

- pour le nombre de cæca: $\mathrm{Y}_{i j k}=\mu+\mathrm{A}_{i}+\mathrm{B}_{j}+\mathrm{C}_{i j}+\mathrm{R}_{i j k}$

— pour le volume de 1'œuf : $\mathrm{V}_{i j}=\mu^{\prime}+\mathrm{A}^{\prime}{ }_{i}+\mathrm{B}^{\prime}{ }_{j}+\mathrm{C}^{\prime}{ }_{i j}$ dans lesquels :

$\mathrm{Y}_{i j k} \quad$ est la valeur transformée du nombre de cæca chez l'individu $k$ de la famille issue du père $i$ et de la mère $j$ dans une série quelconque.

$V_{i j} \quad$ est l'estimation moyenne du volume de l'œuf dans cette famille,

$\mu$ et $\mu^{\prime} \quad$ sont les espérances de ces caractères pour la série considérée,

$\mathrm{A}_{i}$ et $\mathrm{A}^{\prime}{ }_{i}$ sont les effets paternels, $\mathrm{N}\left(\mathrm{O}, \gamma_{\mathrm{A}}\right)$ et $\mathrm{N}\left(\mathrm{O}, \gamma_{\mathrm{A}^{\prime}}\right)$,

$\mathrm{B}_{j}$ et $\mathrm{B}^{\prime}{ }_{j}$ sont les effets maternels, $\mathrm{N}\left(\mathrm{O}, \gamma_{\mathrm{B}}\right)$ et $\mathrm{N}\left(\mathrm{O}, \gamma_{\mathrm{B}^{\prime}}\right)$,

$\mathrm{C}_{i j}$ et $\mathrm{C}^{\prime}{ }_{i j}$ sont les effets d'interaction, $\mathrm{N}\left(\mathrm{O}, \gamma_{\mathrm{C}}\right)$ et $\mathrm{N}\left(\mathrm{O}, \gamma_{\mathrm{C}^{\prime}}\right)$, et

$\mathrm{R}_{i j k} \quad$ est l'écart à la moyenne familiale, $\mathrm{N}\left(\mathrm{O}, \gamma_{\mathrm{R}}\right)$.

Les méthodes d'analyse de variance (modèle factoriel à effets aléatoires) et de covariance sont décrites par SNEDECOR et Cochran (I967). L'estimation de 1'héritabilité est traitée par BECKER ( 1967 ) d'après le modèle classique de FALCONER (Ig60). En supposant l'absence d'épistasie, les composantes de 1a variance relative au nombre de cæca s'interprètent de la façon suivante :

$\gamma_{\mathrm{A}}=\frac{\mathrm{I}}{4} \sigma_{\mathrm{A}}^{2}$

$\gamma_{B}=\frac{I}{4} \sigma^{2}{ }_{A}+\sigma_{M}^{2}$

$\gamma_{\mathrm{C}}=\frac{\mathrm{I}}{4} \sigma^{2}{ }_{\mathrm{D}}$

$\gamma_{\mathrm{R}}=\frac{\mathrm{I}}{2} \sigma_{\mathrm{A}}^{2}+\frac{3}{4} \sigma_{\mathrm{D}}^{2}+\sigma_{\mathrm{E}}^{2}$
$\left(\sigma_{\mathrm{A}}^{2}=\right.$ variance génétique additive $)$,

$\left(\sigma^{2} \mathrm{M}=\right.$ variance de l'effet maternel: aptitudes maternelles),

$\left(\sigma^{2} \mathrm{Y}\right)=$ variance de dominance),

$\left(\sigma^{2} E=\right.$ variance de l'effet d'environnement individuel et de l'erreur de mesure).

Les calculs d'erreur d'estimation sont tirés de BECKER ( 1967 ). Signalons enfin que le test G (SoKAL et ROHLF, I969) a été, par ailleurs, utilisé pour comparer globalement les distributions de cæca des deux sexes. 


\section{Résultats}

\section{Distribution des données}

L'histogramme des nombres de cæca des $75^{\circ}$ individus étudiés est représenté sur la figure I. Les valeurs extrêmes sont 3 I et 95 cæca. La moyenne est 50,50, l'écart-type 7,54 et le coefficient de variation est de $14,94 \mathrm{p}$. Ioo. Cette distribution est asymétrique, de type log-normal. L'analyse des coefficients de Pearson (tabl. I)

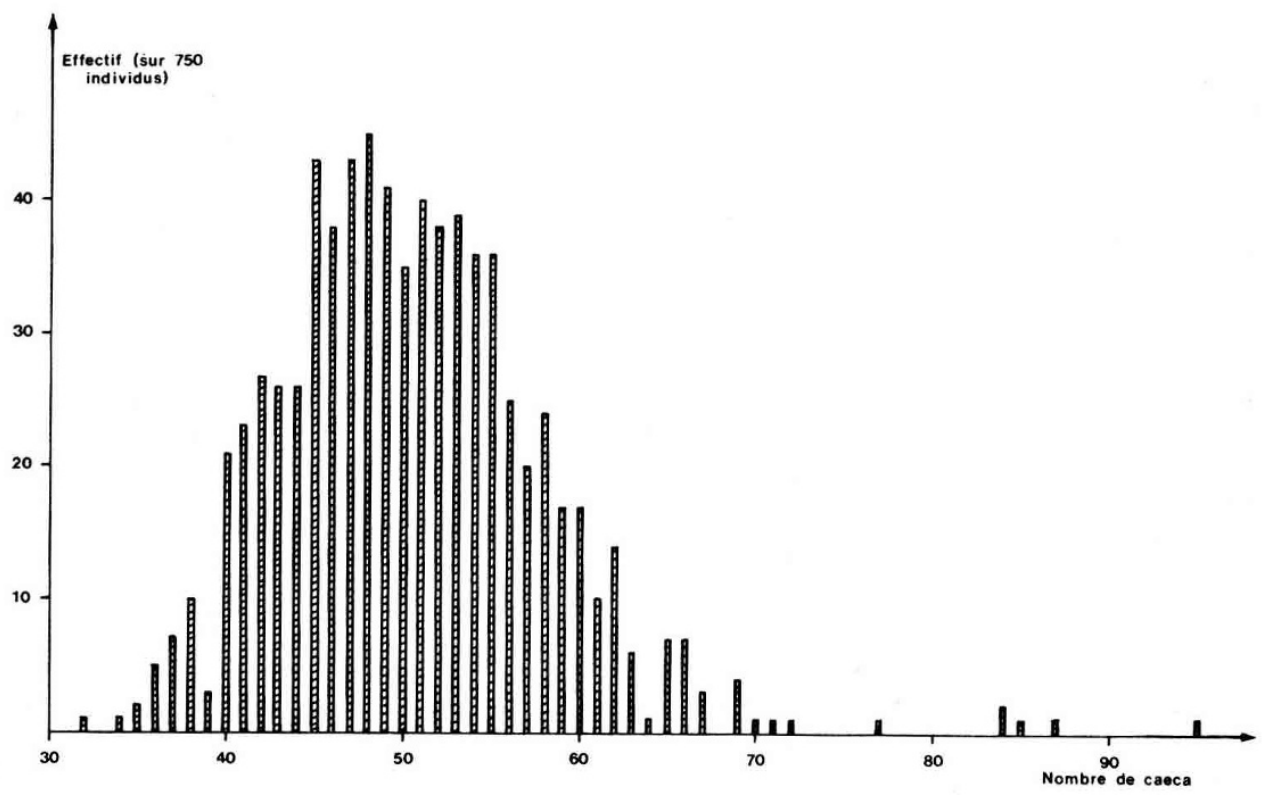

FIG. I. - Distribution du nombre de caca

Distribution of caeca number.

\section{TABLEAU I}

Analyse de la distribution du nombre de coca (X)

Analysis of the distribution of caeca number

\begin{tabular}{|c|c|c|c|c|c|c|c|c|}
\hline & & & \multicolumn{3}{|c|}{ Coefficient d'asymétrie } & \multicolumn{3}{|c|}{ Coefficient d'aplatissement } \\
\hline & & & Estimation & Écart-type & Signification & Estimation & Écart-type & Signification \\
\hline $\begin{array}{r}X \\
\log X\end{array}$ & . & . & $\begin{array}{l}0,977 \\
0,286\end{array}$ & $\begin{array}{l}0,089 \\
0,089\end{array}$ & $\begin{array}{lll}S & \text { I } & \% \\
S & \text { I } & \%\end{array}$ & $\begin{array}{l}3,108 \\
0,812\end{array}$ & $\begin{array}{l}0,179 \\
0,179\end{array}$ & $\begin{array}{lll}S & \text { I } & \% \\
\text { S } & \text { I } & \%\end{array}$ \\
\hline
\end{tabular}


montre que la transformation logarithmique des données a pour effet de réduire l'écart par rapport à une distribution normale, mais de façon très imparfaite. Dans les analyses de variance et de covariance, nous nous référons néanmoins à la variable transformée $\mathrm{Y}=$ Ioo $\log _{10} \mathrm{X}$.

\section{Comparaison des sexes}

La population comprend 384 mâles et 366 femelles, La comparaison des distributions des cæca pyloriques chez les mâles et chez les femelles par un test $G$ ne permet pas de mettre en évidence de variation due au sexe $(\mathrm{G}=38,87$ à 29 degrés de liberté, non significatif).

\section{Comparaison des moyennes de séries}

Nous avons analysé la variation entre séries en utilisant une décomposition hiérarchique dates-séries intradates-familles intraséries. Les résultats (tab1. 2) révèlent un écart significatif à ro $p$. Ioo entre les dates et significatif à I p. roo entre les séries d'une même date. Ce deuxième test utilise cependant une variance résiduelle sous-estimée, dans la mesure où il suppose l'indépendance entre les 25 familles de chaque série.

Du fait des écarts significatifs interséries, l'analyse génétique n'a été réalisée qu’à partir de la variation phénotypique intrasérie.

TABLEAU 2

Analyse de la variation entre séries

Analysis of between series variation

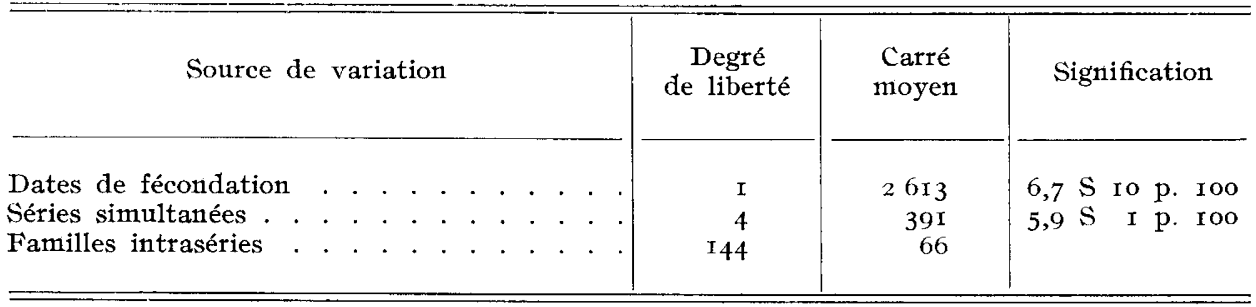

\section{Effet direct du génotype}

L'analyse de la variation intra-série est présentée dans le tableau 3. Pour chaque source de variation, les carrés moyens obtenus dans les 6 séries ne montrent pas d'hétérogénéité significative, à l'exception de la variation résiduelle. Au niveau de cette dernière, l'hétérogénéité provient de 4 familles, d'ailleurs apparentées au sein de la série $n^{0} 5$, qui présentent des variances anormalement élevées.

En dépit de cette irrégularité, l'analyse globale effectuée met en évidence des effets significatifs, y compris l'interaction $\mathrm{P} \times \mathrm{M}$. Lra différence entre les carrés moyens d'origines paternelle et maternelle, par contre, n'est pas significative. 
TABLEAU 3

Analyse de la variation intra-sévie

Analysis of intra-series variation

\begin{tabular}{|c|c|c|c|c|c|c|c|}
\hline \multirow{2}{*}{$\begin{array}{c}\text { Source } \\
\text { de } \\
\text { variation }\end{array}$} & \multirow{2}{*}{$\begin{array}{c}\text { No } \\
\text { série }\end{array}$} & \multicolumn{3}{|c|}{ Carré moyen ( $\mathrm{d}^{0}$ de lib.) } & \multirow{2}{*}{$\begin{array}{c}\text { Test } \\
\mathrm{F}\end{array}$} & \multirow{2}{*}{$\begin{array}{l}\text { Compo- } \\
\text { sante } \\
\text { de } \\
\text { variance }\end{array}$} & \multirow{2}{*}{$\begin{array}{l}\text { (Écart- } \\
\text { type } \\
\text { d'erreur) }\end{array}$} \\
\hline & & $\begin{array}{c}\text { Par } \\
\text { 'série }\end{array}$ & $\begin{array}{c}\text { Test } \\
\text { d'hétéro- } \\
\text { généité }\end{array}$ & Einsemble & & & \\
\hline Pères & 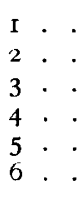 & $\begin{array}{r}\text { I } 70(4) \\
45 \\
\text { I } 20 " \\
\text { I5 } \\
53 " \\
\text { I5 " }\end{array}$ & NS & I I $6,1 \quad(24)$ & S I \% & 3,2 & $(\mathrm{r}, 3)$ \\
\hline Mères & $\begin{array}{ll}\text { I } & : \\
2 & : \\
3 & : \\
4 & : \\
5 & : \\
6 & .\end{array}$ & $\begin{array}{rl}465 & (4) \\
\text { I } 70 " 1 \\
\text { I } 60 " \\
83 & " 1 \\
22 & " 1 \\
45 & " 1\end{array}$ & NS & I $50, I^{\prime}(24)$ & S I $\%$ & 4,5 & $(\mathrm{r}, 7)$ \\
\hline $\mathrm{P} \times \mathrm{M}$ & 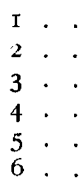 & $\begin{array}{l}46 \text { (I } 6) \\
37 " \\
28 \Rightarrow \\
417 \\
46 " 1 \\
20 "\end{array}$ & NS & $3^{6,2}(96)$ & $S$ I $\%$ & $2, \mathrm{I}$ & $(\mathrm{r}, \mathrm{I})$ \\
\hline Résidus & 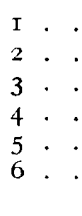 & $\begin{array}{l}20 \text { (I } 00 \\
24 " \\
21 \text { " } \\
19 \text { " } \\
47 " \\
25 "\end{array}$ & $S$ I \% & $25,9(600)$ & & 25,9 & $(\mathrm{r}, 5)$ \\
\hline
\end{tabular}

Variance phénotypique intra-série . . . . . . . . 35,7

Ces résultats conduisent à la décomposition suivante :

$\sigma^{2}{ }_{\mathrm{A}}=\mathrm{I} 2,8$

$\sigma^{2}{ }_{D}=8,3$ si l'on suppose l'absence d'effet d'environnement collectif.

$\sigma^{2}{ }_{\mathrm{M}}=\mathrm{I}, 3$ (ne différant pas significativement de zéro).

$\sigma^{2} \mathrm{E}=\mathrm{I} 3,3$

d'où l'héritabilité : $h^{2}=0,36$ (écart-type d'estimation: 0,15 ). 


\section{Effet de la taille des ceuts}

La taille moyenne des œufs ayant été estimée pour chaque famille par une mesure unique du volume de Ioo œufs, il n'est pas possible d'appréhender les effets individuels intra-lot. L'analyse de la covariance est donc réalisée sur la base des moyennes familiales (tabl. 4).

La variation intra-série du volume $\left(\mathrm{mm}^{3}\right)$ des œufs se décompose en un effet

\section{TABLEAU 4}

Analyse de covariance du volume moyen de l'ouf et du nombre de caca Covariance analysis for the average volume of the egg and the number of caca

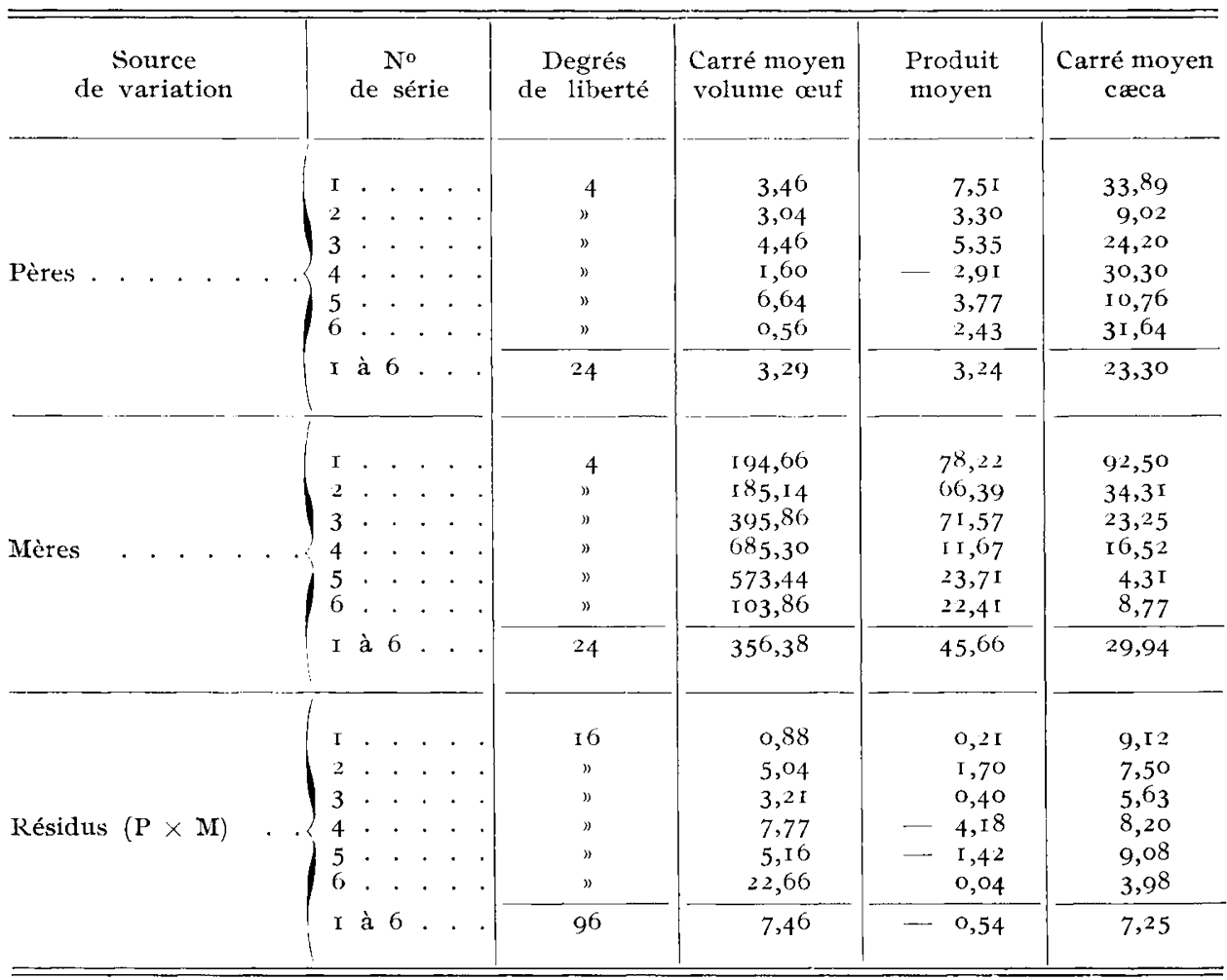

maternel important et un résidu qui recouvre à la fois l'effet de l'échantillonnage et l'erreur de mesure. La variation entre séries, testée par rapport au carré moyen maternel, apparaît inexistante $\left(\mathrm{F}_{(5 ; 24)}=\mathrm{I} 92,63 / 356,38=0,54 \mathrm{I}\right.$, NS). La covariance entre le volume des oufs et le nombre de cæca se situe essentiellement au niveau de la variation maternelle. Dans une représentation graphique des 30 points (5 par série) obtenus à partir des moyennes maternelles (fig. 2), l'effet de la taille des œufs sur le logarithme du nombre de cæca apparaît d'autant plus marqué que la moyenne des cæca est plus faible pour la série considérée. Néan- 


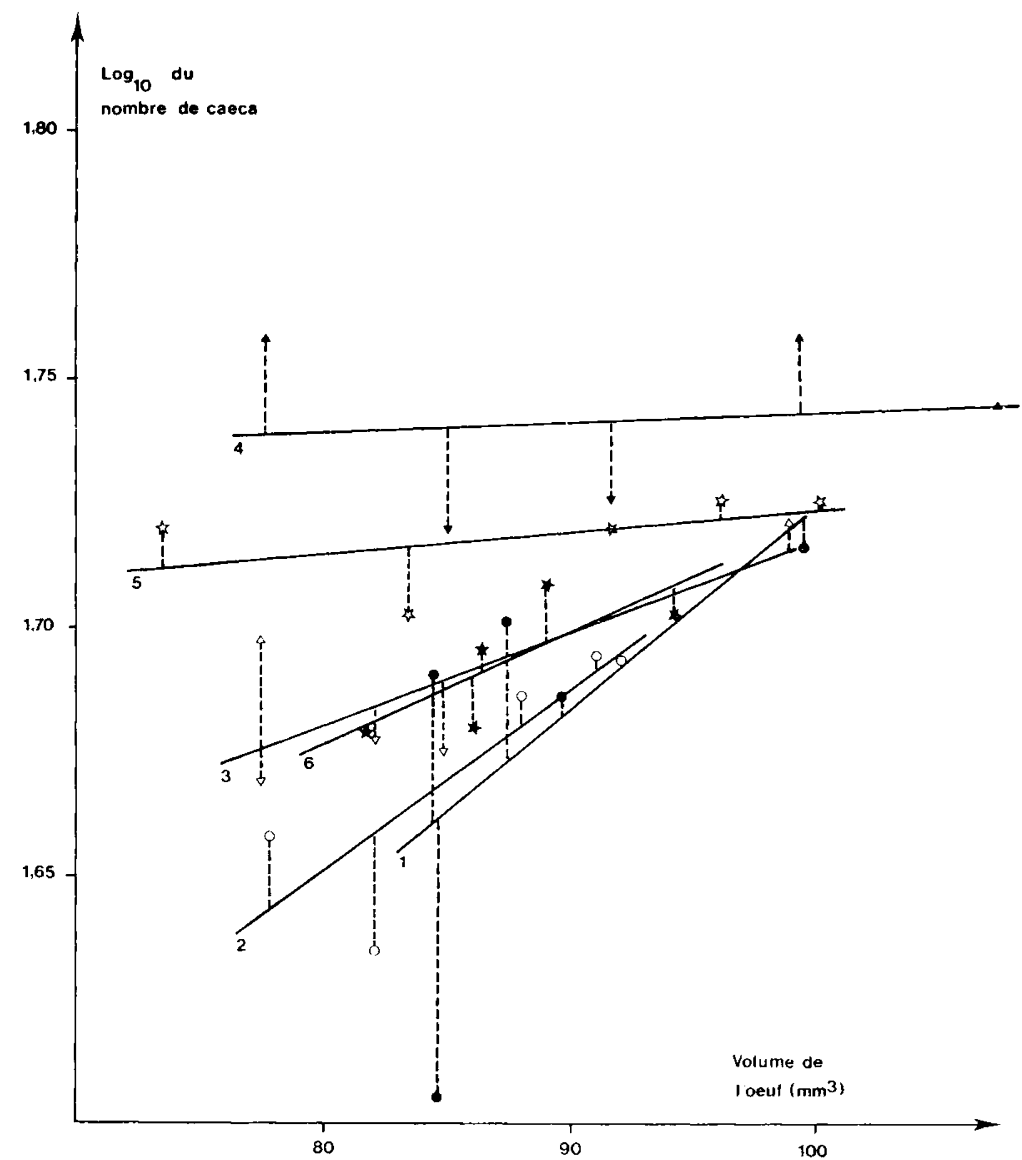

FIG. 2. - Régression du nombre de coeca sur la taille des aufs (moyennes par mère) dans les 6 séries. Regression of number of caeca upon size of eggs (average by mother) in the six series

\section{TABIEAU 5}

Corrélations avec la taille des ouls: résultats moyens intra-série

Correlation with the size of eggs: average intra series results

\begin{tabular}{|c|c|c|c|c|c|}
\hline \multirow{2}{*}{$\begin{array}{l}\text { Source } \\
\text { de variation }\end{array}$} & \multirow{2}{*}{$\begin{array}{l}\text { do de } \\
\text { liberté }\end{array}$} & \multicolumn{2}{|c|}{ Composantes de variances } & \multirow{2}{*}{$\begin{array}{l}\text { Composante } \\
\text { de covariance }\end{array}$} & \multirow{2}{*}{ Corrélation } \\
\hline & & Volume œufs & Cæca & & \\
\hline Pères . . . . & 24 & $-0,8(0,4)$ & $3,2(I, 3)$ & $0,76(0,50)$ & NS \\
\hline Mères . . . . & 24 & $70,0 \quad(19,8)$ & $4,5(1,7)$ & $9,24(4,43)$ & $0,52 \& 5 \%$ \\
\hline Résiduelle . . & 96 & $6,6(0,8)$ & $7,2 \quad(r, 0)$ & $\ldots 0,54(0,70)$ & $=0,08 \mathrm{NS}$ \\
\hline Totale. . . & & 76,6 & I 4,9 & 9,46 & 0,55 \\
\hline
\end{tabular}

N.B. : Ires écarts-types d'erreur approximatifs sont indiqués entre parenthèses. 
moins, le test de parallélisme effectué entre les régressions d'origine maternelle des 6 séries donne un résultat non significatif, de même que celui effectué entre les régressions d'origine résiduelle.

Par suite, une analyse globale intra-série peut être effectuée (tabl. 5). Elle met en évidence une corrélation moyenne d'origine maternelle de 0,52 , significative à $\mathrm{P}=0,05$, les autres composantes de covariance étant négligeables. Ce résultat permet d'estimer, dans l'analyse de la variation des cæca, la part de la composante maternelle de variance due à la taille des œufs : cette composante est d'environ $I, 2$, valeur très voisine de la variance de l'effet maternel estimée plus haut $(I, 3)$.

\section{Discussion et conclusion}

La présente étude permet de confirmer et de préciser certains résultats antérieurs concernant les paramètres phénotypiques et génétiques du nombre de cæca pyloriques chez la Truite fario. Elle soulève par ailleurs un problème nouveau relatif aux effets maternels.

\section{Estimation de la part génétique de variabilité}

La variabilité phénotypique de la population étudiée (coefficient de variation I5 p. IOo) ainsi que la part de cette variabilité attribuable aux facteurs génétiques additifs (héritabilité approximative 0,4 ) sont en accord avec les résultats obtenus antérieurement chez la Truite fario (BERGOT et al., I976) et chez la Truite arc-enciel (BERGot et al., I976; Chevassus et al., I979). Nos estimations sont néanmoins entachées d'une certaine imprécision due à l'effectif relativement faible des reproducteurs utilisés.

Une grande incertitude subsiste d'autre part quant à l'incidence intra-série des facteurs environnementaux, car notre plan d'expérience confond dans la composante d'interaction $\gamma C$ d'une part les effets génétiques de dominance et d'autre part les effets d'environnement commun. On peut cependant supposer que ces derniers sont de faible importance dans la mesure où :

- les conditions physico-chimiques du milieu sont très homogènes d'un compartiment à un autre au sein de notre installation expérimentale,

- le nombre de cæca pyloriques est fixé au cours des premiers mois de la vie de l'alevin (NORTHCOTE et PATTERSON, I960), alors que les effets "compartiment", observés par ailleurs sur la survie et la croissance de ces animaux (BLANC, I979), sont provoqués par des problèmes sanitaires qui n'interviennent guère avant le mois de mai.

Einfin, l'existence d'un effet "série de fécondation " est d'interprétation délicate car il peut représenter des effets incontrôlés de facteurs physico-chimiques (température, oxygénation de l'eau) de facteurs biologiques (maturité des reproducteurs) ou expérimentaux (manipulation des gamètes, des œufs et des alevins).

\section{Effet maternel inhérent à la taille des ceufs}

Il semble logique d'interpréter la corrélation observée entre le volume des œufs et le nombre de cæca comme une relation de cause à effet dans laquelle le nombre de cæca constitue la variable dépendante. L'effet de la taille des œufs 
suffit à rendre compte globalement de l'influence maternelle excédant la contribution génétique directe, cette dernière étant estimée par ailleurs à partir de la variance d'origine paternelle.

Mais d'autre part, il apparaît que cette influence est plus marquée dans les croisements où le nombre moyen de cæca est plus faible. L'action conjointe sur les cæca pyloriques de l'effet maternel inhérent à la taille des œufs et des autres effets génétiques et environnementaux ne semble donc pas suivre un modèle entièrement additif, mais plutôt un modèle comportant certains termes interactifs.

Ce problème justifie la recherche d'informations expérimentales supplémentaires sur les effets maternels, et l'essai de méthodes statistiques autres que les méthodes adaptées aux modèles additifs classiques.

Reçu poù publication en 1979.

\section{Remerciements}

L'analyse statistique de nos données a été réalisée avec le matériel de calcul, les programmes et l'aide technique de la Commission de Biométrie du Centre de Recherches de Bordeaux, I.N.R.A.

\section{Summary}

Genetic determinism of the number of pyloric caeca in brown Trout (Salmo trutta) and rainbow Trout (Salmo Gairdneri)

III. - Influence of the genotype and egg size on the realization of the character in brown Trout

An analysis of the variation of the number of pyloric cæca in hatchery-reared brown trout showed that:

- the sex of the animals has no significant influence;

- an important part of variability is caused by additive genetic factors (approximate heritability 0.4$)$;

- there is a maternal effect which inheres to the size of eggs; alevins hatched from large eggs tend to have high numbers of caeca (correlation +0.5 );

- lastly, the joined action of that maternal factor and other genetic and environmental factors apparently includes interactive effects; further studies on that point would be useful.

\section{Références bibliographiques}

BECKER W. A., I967. Manual of procedures in quantitative genetics. Washington State Univ. Press (2nd ed.), Pullman, Was., r 30 p.

Bergot P., Blanc J. M., Chevassus B., I979. Relations entre le nombre de caeca et la croissance chez la truite arc-en-ciel. $23^{\mathrm{e}}$ Congr. ann. Ass. Fr. Limnologie, Biarritz. Bull. Cent. Et. Rech. Scient., 12 (3), 534-535.

Bizkgó P., Chenassus B., Blanc J. M., r976. Déterminisme génétique du nombre de caeca pyloriques chez la truite fario (Salmo trutta Linné) et la truite arc-en-ciel (Salmo gairdneri Richardson). I. - Distribution du caractère et variabilité phénotypique intra et interfamilles. Ann. Hydrobiol., 7, I05-II4.

BFRgot P., SOLARI A., LUQUET P., I 975. Dimensions des caeca pyloriques chez la truite arc-enciel. Influence de la taille du poisson et du nombre de caeca. Ann. Hydrobiol., 6, I45-158. 
BLANC J. M., 1979. Effets familiaux sur la croissance d'alevins de Truite Fario (Salmo trutta L.). $23^{\mathrm{e}}$ Congr. ann. Ass. Fr. Limnologie, Biarritz. Bull. Cent. Et. Rech. Scient., 12 (3), 537.

Chevassus B., Blanc J. M., Bergor P., 1979.Déterminisme génétique du nombre de caeca pyloriques chez la truite fario (Salmo trutta Linné) et la truite arc-en-ciel (Salmo gairdneri Richardson). II. - Effets du génotype, du milieu d'élevage et de l'alimentation sur la réalisation du caractère chez la truite arc-en-ciel. Ann. Génét. Sél. Anim., 11, 79-92.

FALCONER D. S., I960. Introduction to quantitative genetics. Oliver and Boyd. Ed. Edinburgh, $365 \mathrm{p}$.

NorthCote T. G., Patrerson R. J., 1960. Relationship between number of pyloric caeca and length of juvenile rainbow trout. Copeia, 3, 248-250.

SNedecor G. W., Cochran W. G., 1967. Statiscal methods. Iowa State Univ. Press, Ames, 593 p.

Sokal R. R., RohlF F. J., 1969. Biometry. Freeman, San Francisco, Cal., 776 p. 